\title{
Effect of Multi Jets on Cavitation Performance of Globe Valves*
}

\author{
Srambickal RAMMOHAN**, Sivasankaran SASEENDRAN** \\ and Sivasailam KUMARASWAMY*** \\ ** Fluid Control Research Institute, Kanjikode West, Palakkad, 678623 India \\ E-mail : s.rammohan@fcriindia.com \\ *** Dept. of Mechanical Engineering, Indian Institute of Technology, Madras, Chennai, India
}

\begin{abstract}
Globe valves are one of the oldest valve types used for throttling applications for all sizes due to better controllability and range. One of the major limitations associated with the use of globe valves in liquid application is cavitation. It takes place both in part open and in fully open conditions due to varied reasons. There are different designs of globe valves available but for control valve applications, cage and plug designs are widely employed. Cage and plug design consists of body, valve cage, plug and an actuating mechanism. Actuating mechanism is connected to the valve plug (moving part), through valve shaft. There are many investigations reported about the flow visualization and numerical simulation of normal type globe valves. But study on valves with cage and plug design are not tried in detail. The objective of the present work is to provide a comprehensive study of flow through a globe valve with cage and plug design with emphasis on cavitation. Cavitation reduction is achieved by breaking the flow in the form of more than one liquid jets, there by increasing the turbulence in the valve flow path. This ensures the local static pressure not going below vapour pressure. Experimental studies were done in the water test facility with an operating pressure of 1.6 MPa and flow rate of $0.05 \mathrm{~m}^{3} / \mathrm{s}$. In the study, total area of opening of the valve and the valve stroke were kept constant.
\end{abstract}

Key words: Cavitation, Globe Control Valve, Plug and Cage Design, Experimental Technique, Jet Configuration

\section{Introduction}

Valves are widely used in irrigation, energy, water distribution networks and process industries and in many other areas. Among the different types of valves used in the process industry, control valves play a vital role in the functioning and profitability of a plant. Trouble-free operation of control valve in the piping network is essential to avoid a situation leading to the total closure of the concerned industrial activity. Further, their efficient working leads to an effective use of the available resources. The abundant improvements in the design and performance of control valves are still insufficient to claim perfection in the agreement of theory and practice. The phenomenon of cavitation in control valves is the one in which some more progress can be achieved.

Globe valves are widely used for throttling applications in the process industry for both liquid and gaseous applications. The main advantages are relatively low cost, linear characteristics and good controllability and range. To obtain the required flow and pressure drop characteristics for the valves, different types of internals were evolved for globe type valves. Cage and plug internal is one among them. One of the major limitations associated with the use of globe valves in liquid application is cavitation. This limits the 
operating regime of valves. To combat cavitation in valves, valve manufactures have evolved different solutions including the design improvement, use of harder materials to reduce erosion rate, limiting the valve operation to some critical values so that downstream pressure never goes below vapour pressure etc.

In the succeeding paragraphs experiments conducted on a $75 \mathrm{~mm}$ nominal bore globe type control valve are described. Test valve is with cage and plug design suitable for cavitation-free applications. Effect of making the water jet coming out of the valve vena contracta into a combination of smaller jets was tried. The effect on the flow capacity and the cavitation behavior is studied in detail.

Hammitt $^{(1)}$ described in detail about the bubble growth and nucleation studies conducted on orifice, nozzles and valves. He has concluded that basic flow pattern is more complex in case of valves as compared to orifices \& nozzles; less basic understanding exists on bubble growth as part of cavitation. Brennen ${ }^{(2)}$ describe modeling of a one-dimensional flow through a convergent divergent nozzle with vapour bubble present. Here a two-phase flow through the nozzle from entry itself was considered. In the case of cavitation in valves, the numerical models of Wang \& Brennen ${ }^{(3)}$ and David \& Stewart ${ }^{(4)}$ can be combined with two-phase flow starting after the convergent section of the nozzle. The starting of the flow is to be initiated by static pressure going below a threshold value. This value can be correlated to the local static pressure experienced in the valve. Ramamurthi \& Nandakumar ${ }^{(5)}$ studied characteristics of flow in the separated, attached and cavitated flow of small sharp edged cylindrical orifices. They have reported that onset of cavitation observed was dependent on the diameter and aspect ratio of the orifices under study. They have studied on orifice plates with diameters varying from 0.3 to $2 \mathrm{~mm}$. This study was extended by Ramamurthy ${ }^{(6)}$ and Patnaik to investigate the effect of periodic disturbance present in the flow on the inception of cavitation. Cavitation characteristics of orifices were studied by Takahashi ${ }^{(7)}$ et al. The spatial distribution of cavitation pressure down stream of the orifice along the pipe line was studied. Cavitation of butterfly valve downstream of a multi holed orifice was also investigated in this study.

NUREG report of Tullis ${ }^{(8,9,10)}$ describes the cavitation tests performed in different types of orifice plates. It was reported that the results of the multi holed orifice plate tests can be extrapolated for control valves with reasonable accuracy. They have generated typical graphs showing the intensity of cavitation as a function of the ratio of upstream to downstream pressure in valves. Cudina ${ }^{(11)}$, describes the detection of cavitation in centrifugal pumps with noise signals. He employed audible noise as a measure of cavitation. Gudra $^{(12)}$ and Opulinski in their paper described how the analysis can be improved if Cepstrum analysis is employed. This will enable more lucid interpretation of cavitation signals.

\section{Nomenclature}

\begin{tabular}{|c|c|c|}
\hline \multicolumn{2}{|c|}{$\mathrm{P}_{\mathrm{in}}, \mathrm{P}_{1}$ Inlet pressure of valve } & $(\mathrm{kPa})$ \\
\hline \multicolumn{2}{|c|}{$\mathrm{P}_{\mathrm{dn}}, \mathrm{P}_{2}$ Outlet pressure of valve } & $(\mathrm{kPa})$ \\
\hline $\mathrm{P}_{\mathrm{v}}$ & Liquid vapour pressure & $(\mathrm{kPa})$ \\
\hline $\mathrm{Cv}$ & Valve capacity - Q / DP ${ }^{0.5}$ & $\left(\mathrm{~m}^{3} / \mathrm{h} /\left(\mathrm{kg} / \mathrm{cm}^{2}\right)^{5}\right.$ \\
\hline $\mathrm{r}_{\mathrm{h}}$ & Hydraulic radius & $(\mathrm{mm})$ \\
\hline$\rho$ & Fluid Density & $\left(\mathrm{kg} / \mathrm{m}^{3}\right)$ \\
\hline $\mathrm{d}$ & Width of hole & $(\mathrm{mm})$ \\
\hline $\mathrm{h}$ & Height of hole & $\mathrm{mm})$ \\
\hline $\mathrm{D}$ & Pipe diameter & $(\mathrm{mm})$ \\
\hline Q & Flow rate & $\left(\mathrm{m}^{3} / \mathrm{h}\right)$ \\
\hline $\mathrm{DP}$ & Differential pressure across test valve & $\left(\mathrm{kg} / \mathrm{cm}^{2}\right)$ \\
\hline$\sigma$ & Cavitation index & $\left(\mathrm{P}_{\text {in }}-\mathrm{P}_{\mathrm{v}}\right) /\left(\mathrm{P}_{\text {in }}-\mathrm{P}_{\text {out }}\right)$ \\
\hline$\sigma_{a}$ & Critical cavitation index (vibration) & \\
\hline
\end{tabular}




\section{$\sigma_{d} \quad$ Critical cavitation index (Dynamic pressure)}

\section{Details of Test valve}

The type of valve used for the study was a normal $75 \mathrm{~mm}$ NB Globe type control valve with cage and plug design. These types of valves are prone for cavitation in the liquid applications. Figure 1 shows the details of valve tested. A valve cage with 4 holes drilled, combination plug and body dimensions are shown. The diameter of the holes drilled were $17.5 \mathrm{~mm}$ with an effective total flow area of $962.11 \mathrm{~mm}^{2}$. The valve plug is connected to a pneumatic actuator and manual option for position control. The valve is connected to the test setup piping through flanges. The inner diameter of the pipe and the inner diameter of the valve were same at the inlet and outlet. Valve was mounted with an Upstream and downstream lengths of $750 \mathrm{~mm}$ and $1500 \mathrm{~mm}$ corresponding to 10 and 20 diameters of the pipe. This was selected so that the flow is fully developed at the valve inlet and full pressure recovery takes place in the pipe after the valve under study.

$\mathrm{P}_{\text {in }}\left(\mathrm{P}_{1}\right)$ is the stagnation pressure at the inlet of the pipe. $\mathrm{P}_{\text {out }}\left(\mathrm{P}_{2}\right)$ is the static pressure at 20 pipe diameters downstream of test valve. During experimental trials, the static pressure $\mathrm{P}_{2}$ was kept constant with varying inlet pressure $\mathrm{P}_{1}$ and flow rate.
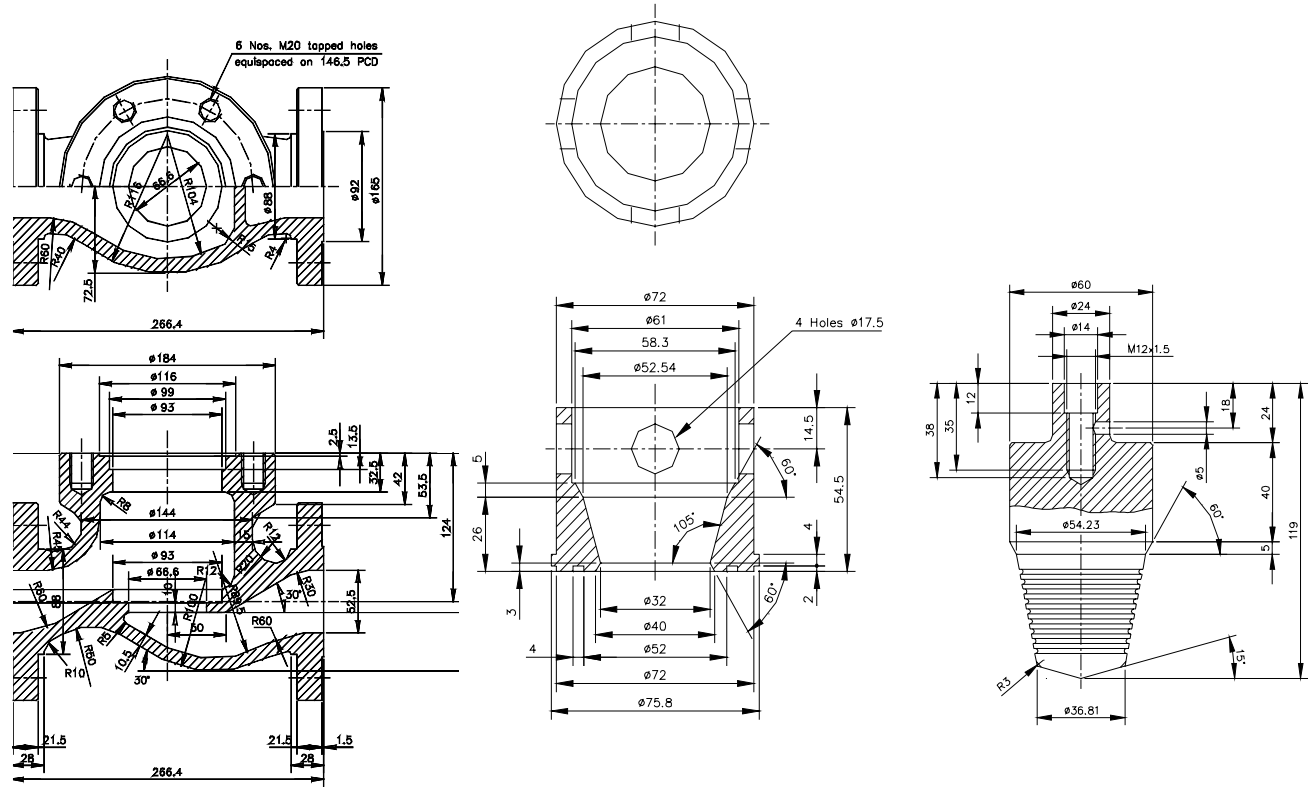

Fig. 1 Valve plug and cage with 4 holes

The fluid flowing through the pipe was water with density $(\rho)$ of $998.5 \mathrm{~kg} / \mathrm{m}^{3}$ and dynamic viscosity $(\mu)$ of $1.12 * 10^{-3} \mathrm{Ns} / \mathrm{m}^{2}$.

Table 1 Hole configuration of cages

\begin{tabular}{|c|c|c|c|c|c|c|}
\hline $\begin{array}{l}\text { Sl. } \\
\text { No }\end{array}$ & $\begin{array}{l}\text { No of } \\
\text { holes }\end{array}$ & $\begin{array}{l}\text { Width of each hole } \\
\text { (d) in mm }\end{array}$ & $\begin{array}{c}\text { Total area } \\
\left(\mathrm{mm}^{2}\right)\end{array}$ & $\begin{array}{l}\text { Height of hole } \\
\text { (h) in mm }\end{array}$ & Layc & ut of holes \\
\hline 1 & 3 & 19.23 & 963.24 & \multirow{5}{*}{17.50} & \multirow{5}{*}{$\mathrm{h}$} & \multirow{4}{*}{$\longleftarrow$ d } \\
\hline 2 & 4 & 17.5 & 962.11 & & & \\
\hline 3 & 6 & 10.5 & 960.54 & & & \\
\hline 4 & 8 & 7.60 & 964.84 & & & \\
\hline 5 & 12 & 4.87 & 961.62 & & & \\
\hline
\end{tabular}


Five different configurations of the cages were tried during the study. The effect of breaking total flow area into jets of smaller sizes, thereby decreasing the mixing length of jets and increasing the minimum static pressure developed at the outlet of the valve cage were studied. Configurations tried on the cage were with 3, 6, 8 and 12 holes of width(d) machined on the cage apart from 4 jet configuration in the original case. In all cases, the total jet area was kept nearly constant as $981.11 \mathrm{~mm}^{2}$. Also the height of the jets (h) was kept same in all cases as $17.5 \mathrm{~mm}$. All the jets will be in the form of non-circular jets, except for 4 holed case. Details of hole configuration provided in the cage along with dimensional details are shown in Table 1. Figure 2 shows the photograph of the cages employed during testing program. Same plug was used for all the cages.

In all the above cases the experimentation was done and flow rate for varying differential pressure across the test valve was obtained. The valve downstream pressure was kept constant. Provision was made to mount accelerometer stud on the valve body close to the outlet flange of test valve.

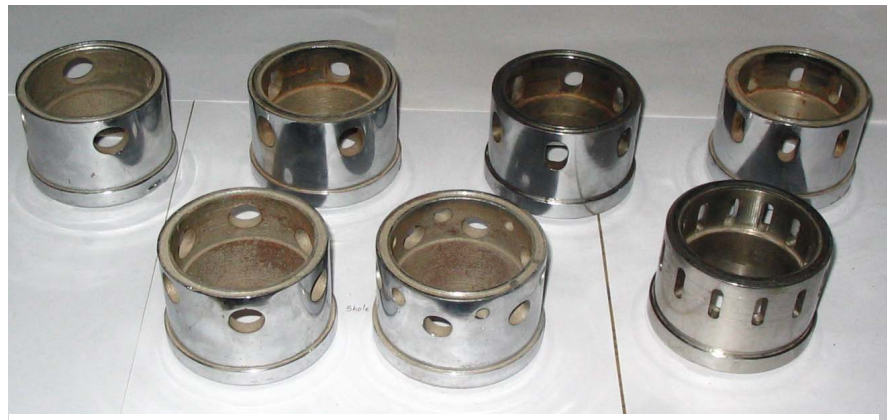

Fig. 2 Photograph of the cages tried

\section{Experimental set up}

A series of experiments were performed on a $75 \mathrm{~mm}$ size globe valve with cage plug details described in $\S 2$. The schematic of the experimental test setup is shown in Fig. 3 . The test setup was designed as per Tullis ${ }^{(9,10)}$. Open loop re-circulating system includes an underground sump that holds about 300 cubic meters of water, a $150 \mathrm{~kW}$, multi stage centrifugal pump and valves to control and bypass the flow.

Water at ambient temperature was pumped with a maximum flow rate of $0.05 \mathrm{~m}^{3} / \mathrm{s}$ at a head of $140 \mathrm{~m}$. Flow rate and static pressure in the test loop were controlled with a by pass

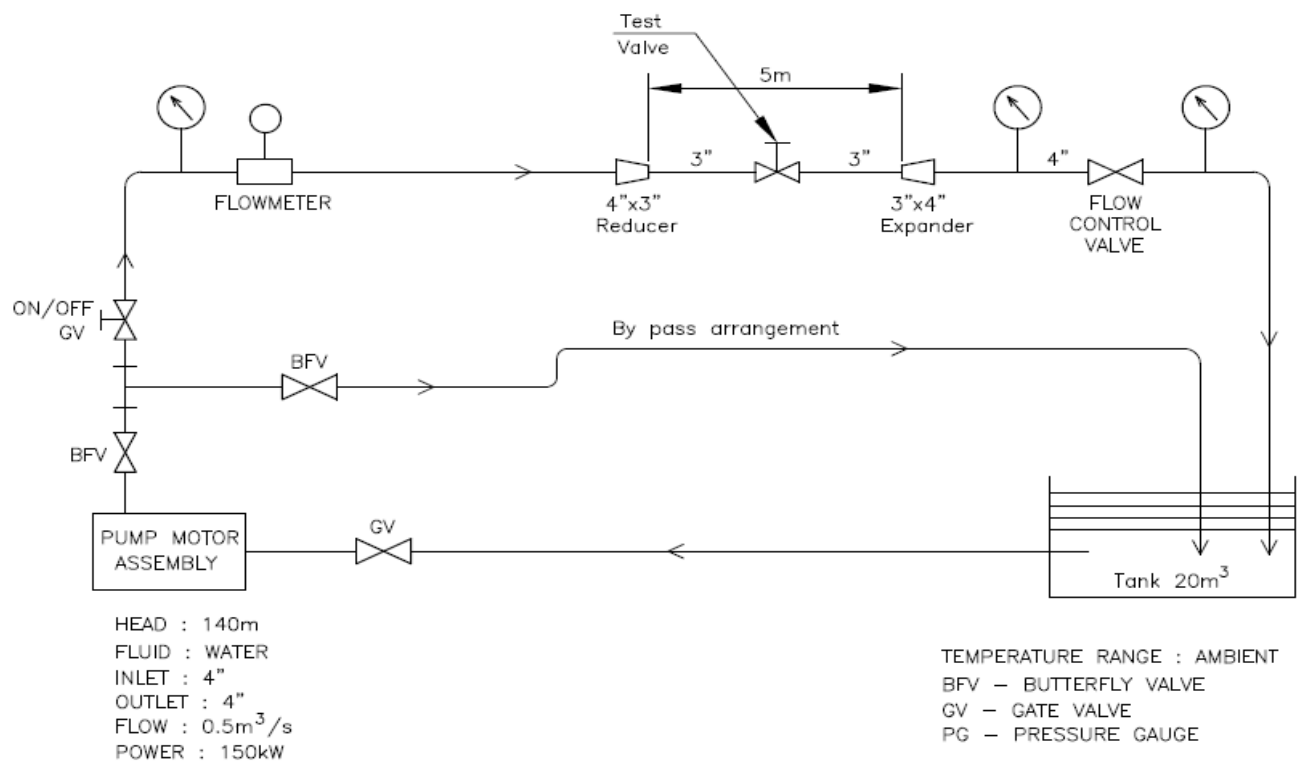

Fig. 3 Schematic of experimental set up 
valve arrangement provided at the discharge side of the pump. Flow rate through the loop was measured with a flow meter $(100 \mathrm{~mm}$ orifice flow meter) provided upstream of the test valve. The outlet of the test valve was connected to the sump with a control valve in the loop. With this outlet pressure of the test valve can be controlled. By adjusting the bypass and control valves provided in the loop, the required differential pressure can be created across the test valve. The test valve opening was controlled by manually opening / closing the valve and measuring the valve stroke with a calibrated dial gauge.

Pressure gauge was used to measure the static pressure at the inlet of the test valve. The pressure/vacuum at the outlet of the test valve was monitored using a compound gauge. A Quartz type differential pressure transducer was used to measure the differential pressure created by test valve. Pressure tapings are provided one pipe diameter (1D) upstream and six pipe diameters (6D) down stream of the test valve. Pressure tapings were also provided at one pipe diameter each, downstream of the test valve up to $750 \mathrm{~mm}$. The pressure drop created by the valve for a specific flow rate at particular valve opening, was determined by measuring the differential pressure across the valve between $1 \mathrm{D} \& 6 \mathrm{D}$ pressure tapings. Calibrated pressure transducer was used for the discharge pressure measurement. Water temperature inside the pipe was measured using Resistance temperature detector. The pressure upstream of the test valve was controlled with the bypass valve provided in the loop. Care was taken to avoid cavitation taking place in the bypass and downstream control valves of the loop.

Table 2 Flow test results

\begin{tabular}{|c|c|c|c|c|}
\hline $\begin{array}{c}\text { Sl. } \\
\text { No. }\end{array}$ & $\begin{array}{c}\text { No. of } \\
\text { holes }\end{array}$ & $\begin{array}{c}\text { Jet width } \\
\text { in mm }\end{array}$ & $\begin{array}{c}\text { Hydraulic Radius } \\
\left(\mathrm{r}_{\mathrm{h}}\right) \text { in mm }\end{array}$ & $\begin{array}{c}\text { Valve } \\
\text { Capacity (Cv) }\end{array}$ \\
\hline 1 & 3 & 19.23 & 4.63 & 33.0 \\
\hline 2 & 4 & 17.50 & 4.38 & 28.2 \\
\hline 3 & 6 & 10.50 & 3.41 & 24.5 \\
\hline 4 & 8 & 7.60 & 2.76 & 26.2 \\
\hline 5 & 12 & 4.87 & 1.99 & 25.7 \\
\hline
\end{tabular}

A quartz type dynamic pressure transducer was placed downstream of the test section to measure the pressure fluctuations in the flow. This was positioned flush with the pipe. Accelerometers were mounted on the test valve and pipe down stream of the test valve to sense vibration caused by cavitation. Mounting was done with suitable studs supplied along with the accelerometer. Fast Fourier Transform (FFT) analysis was performed on the collected signals to determine the dominant frequencies. After the water leaves the test section, it is routed back to the sump. Care was taken to ensure full running of the pipe during experimentation.

A flow meter was used to measure the flow rate through the test valve and is placed upstream of the test valve. An orifice flow meter suitable for the required range of discharge used in these experiments was of $75 \mathrm{~mm}$ nominal diameter and diameter ratio of 0.7 . A differential pressure transmitter was used to measure the pressure difference across the orifice. The uncertainty of the transmitter was better than $\pm 0.5 \%$. Sufficient straight length was provided after the flow meter and the test valve.

\section{Results}

\subsection{Flow tests}

The total area of flow and the height of each opening were maintained constant. The Diameter / width of the holes and the number of holes were varied in the trials. During trials, Performance of the cage was analysed with Valve capacity $(\mathrm{Cv})$ defined as

$$
\mathrm{Cv}=\mathrm{Q} / \mathrm{DP}^{0.5} \text {. }
$$




\section{$\mathrm{Q}$ - Flow in $\mathrm{m}^{3} / \mathrm{h}$ and DP - Differential Pressure across valve in $\mathrm{kg} / \mathrm{cm}^{2}$}

Figure 4 shows the plot of valve capacity $(\mathrm{Cv})$ for various differential pressures across the test valve for 12 holed configuration. As the differential pressure increases, the valve capacity decreases or fluctuates. Similar results were obtained for other hole configurations also.

The mean $\mathrm{Cv}$ obtained for various differential pressures across the valve was computed. The hydraulic radius $\left(r_{h}\right)$ and loss coefficient for different hole configurations of the cage were also shown in Table 2 and Fig. 5

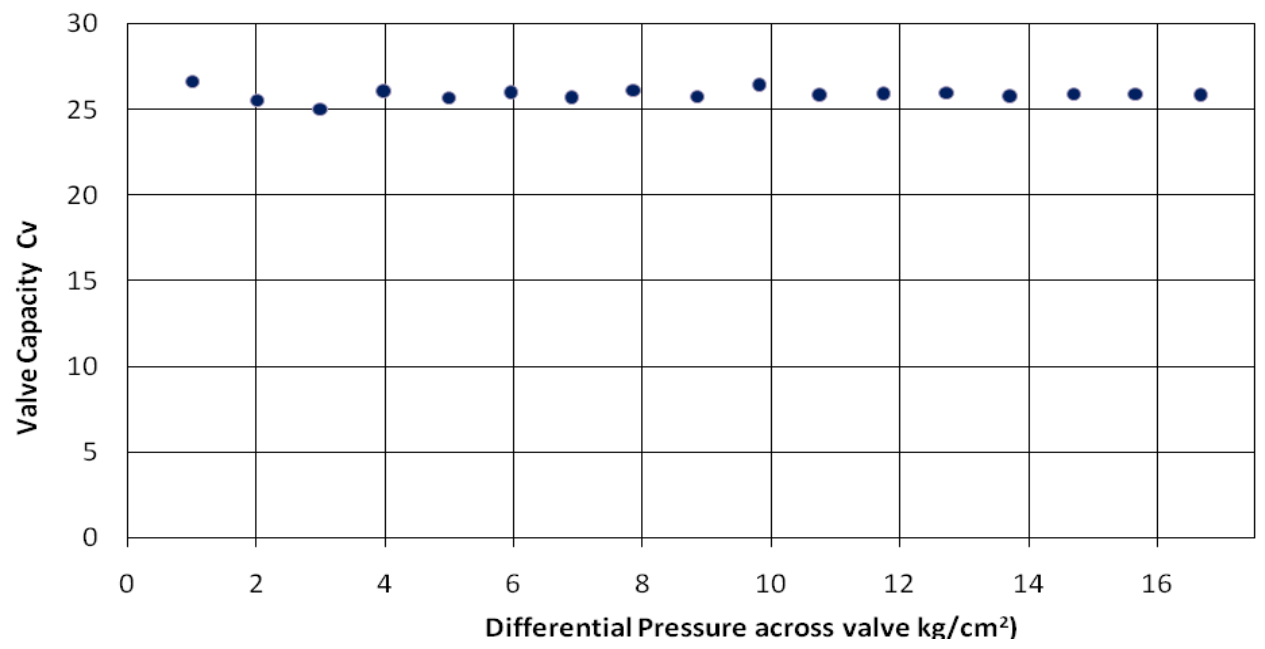

Fig. 4 Plot of valve Capacity $(\mathrm{Cv})$ as a function of $\sigma$ and DP across valve

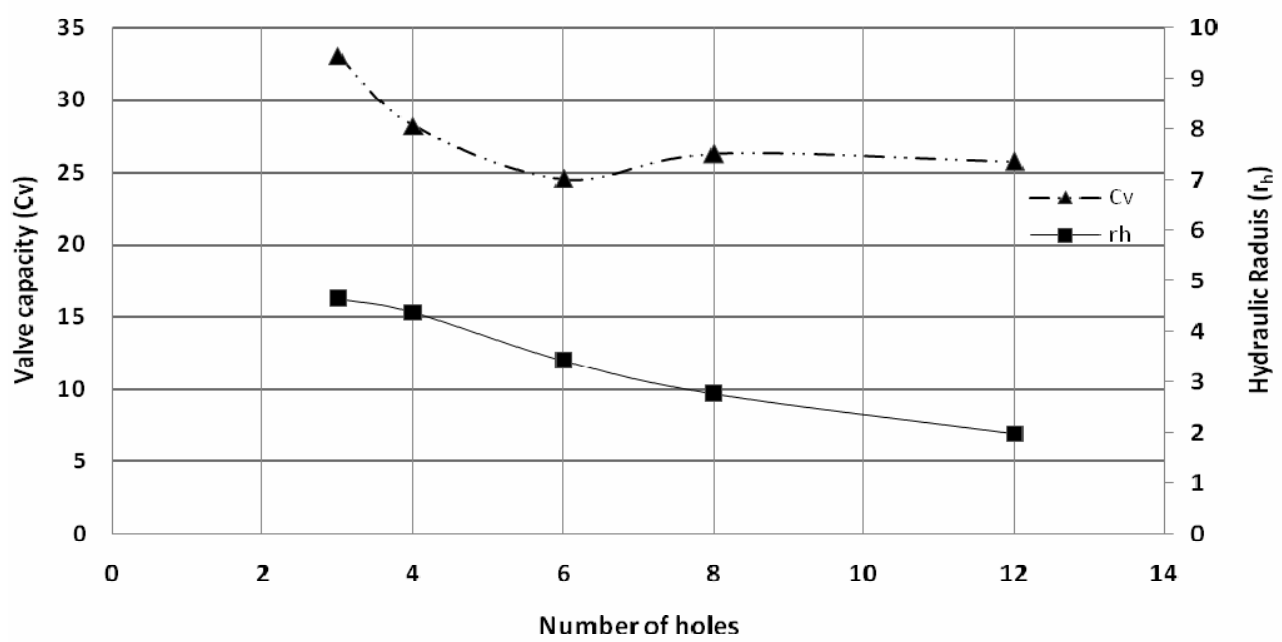

Fig. 5 Plot of $\mathrm{Cv}$ and $\mathrm{r}_{\mathrm{h}}$ as a function of number of holes in the cage

Hydraulic radius $\left(r_{h}\right)$ for different cage configuration were computed as

$\mathrm{r}_{\mathrm{h}}=$ Flow area / Wetted perimeter for a individual holes.

It can be seen that with the number of holes increasing, the $\mathrm{Cv}$ of the valve decreases. This is more evident from Fig.5. It can also be seen that, as the number of holes increases, the decrements in $\mathrm{Cv}$ become less. Also as the width of the jet decreases (with increase in number of holes), the hydraulic radius also decreases.

\subsection{Cavitation tests}

Results of cavitation tests were analysed in two different methods as described below, 


\subsubsection{Analysis of overall values}

Figure 6 shows the effect of various differential pressure across the test valve on the vibration and dynamic pressure fluctuations in the flow. Here the fluid borne noise and pipe wall vibrations downstream of the test valve were measured using a dynamic pressure sensor and accelerometer respectively. The overall Root Mean Square (rms) value of the fluid borne noise and vibration (along $\mathrm{Y}$ axis) are plotted with Sigma (along $\mathrm{X}$ axis) defined as

$$
\text { Sigma }(\sigma)=\left[\left(p_{\text {in }}-p_{v}\right) /\left(p_{\text {in }}-p_{\text {out }}\right)\right]
$$

With reduction in $\sigma$, the rms value of the signal for both vibration and hydraulic noise increases.

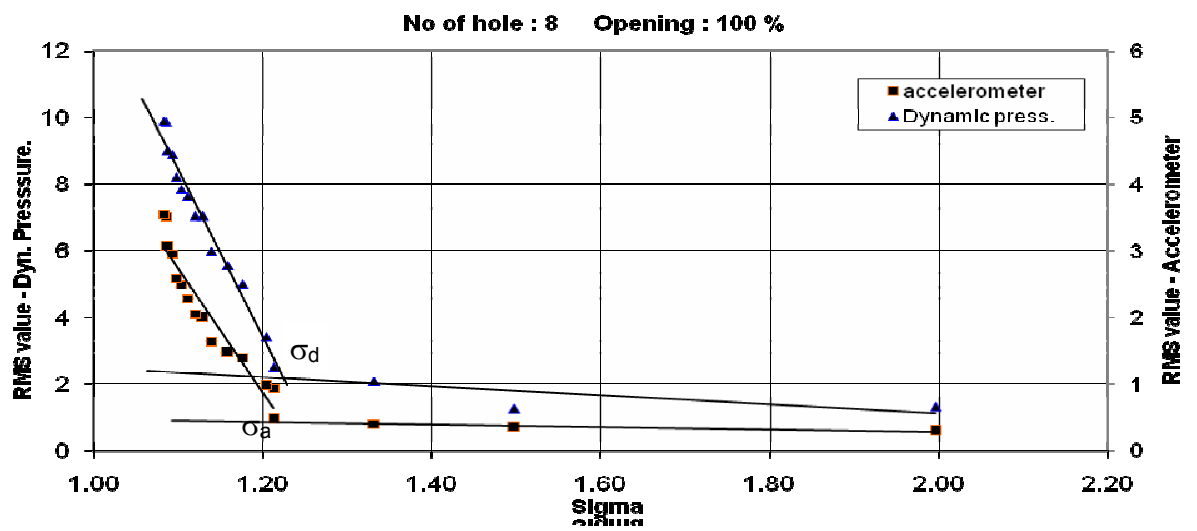

Fig. 6 Plot of overall RMS as a function of sigma \& estimation of critical sigma

It was also observed that irrespective of number of holes, signal strength increases with reduction in $\sigma$ (increase in upstream pressure). It is possible to fit separate straight lines earmarking different zones of cavitation. It can also be observed that both vibration and fluid borne noise (dynamic pressure fluctuations) show similar trend. The intersection of two straight lines is considered as critical $\sigma$. This corresponds to a critical behavior in the cavitation characteristic of the cage. In Fig 6 , ' $\sigma_{a}$ ' refers to critical sigma derived with accelerometer output and ' $\sigma_{\mathrm{d}}$ ' refers to critical sigma derived dynamic pressure sensor output.

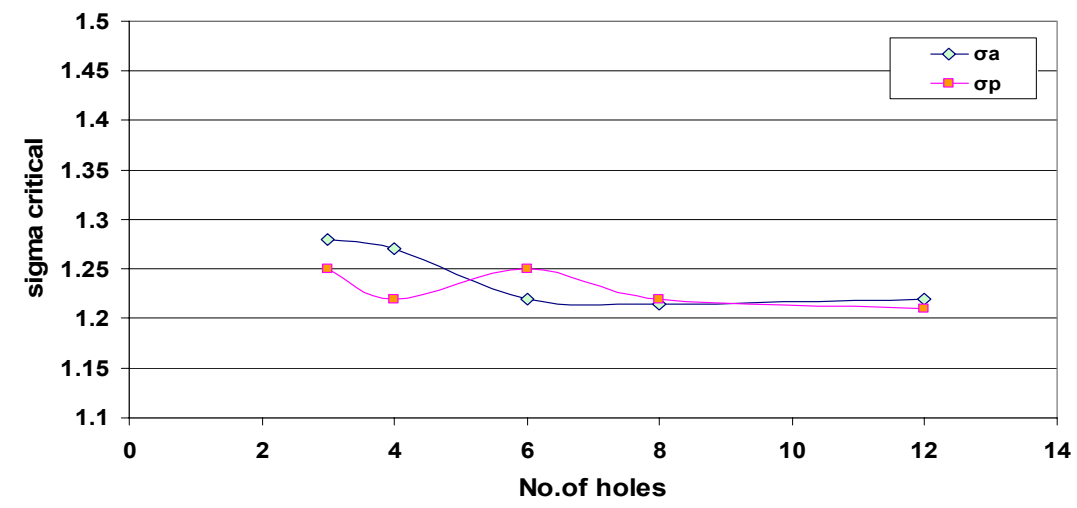

Fig. 7 Effect critical $\sigma$ on number of holes

Figure 7 shows the plot of critical sigma (' $\sigma_{\mathrm{a}}$ ' and ' $\sigma_{\mathrm{d}}$ ') obtained from vibration and dynamic pressure measurements for various cage configurations. From Fig. 7, it can be concluded that vibration data posts a clear trend in the overall value as the number of holes were varied. For further analysis, vibration data was used.

\subsubsection{FFT analysis of data}

To study the cavitation inception and development, the vibration data collected were further analysed using Fast Fourier Transform analysis for frequency spectrum. Figure 8 
shows the frequency spectrum of accelerometer output for various differential pressures across the test valve. The configuration employed for this figure was the cage with 6 holes and signal from accelerometer was analysed. Valve opening was kept full. A lower cutoff frequency of $6 \mathrm{kHz}$ was provided to avoid signals due to mechanical reason.

For a constant valve opening, plot was made for varying differential pressure across the valve $(\sigma)$. The band width (BW) was kept as $1.6 \mathrm{kHz}$ to have a clear frequency spectra. Clear frequencies can be identified with decrease in $\sigma$, the first peak was obtained between $6-12$ $\mathrm{kHz}$.

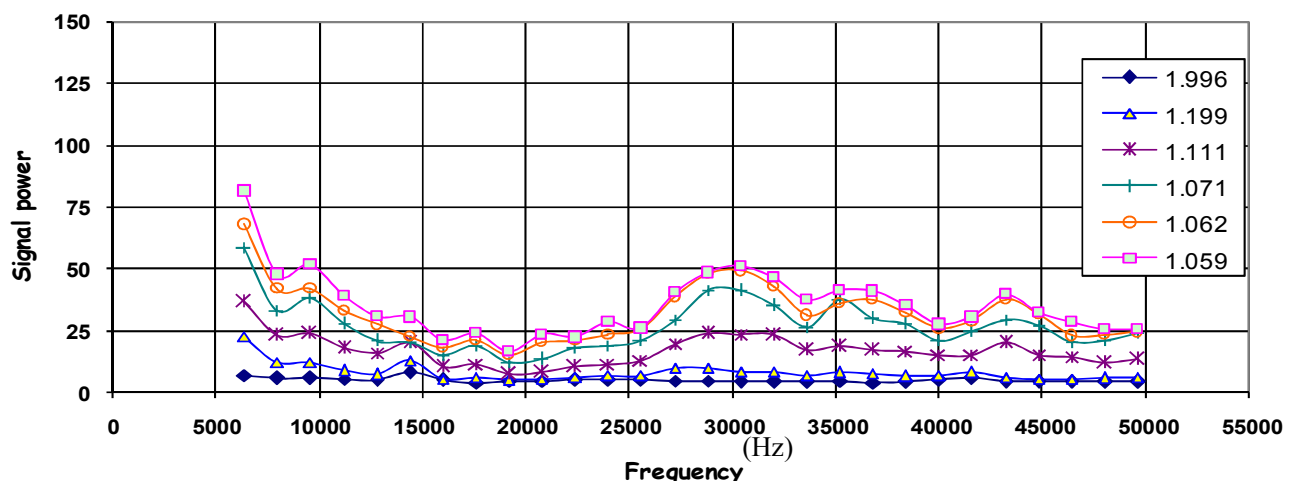

Fig. 8 FFT spectrum of accelerometer above $6 \mathrm{kHz}(1600 \mathrm{~Hz} \mathrm{BW})$ for various $\sigma$

Figure 9 shows the FFT spectrum of the accelerometer output for different cage configurations for 14 bar differential pressure across valve $(\sigma=1.074)$. From a study of Figs. $8 \& 9$ together, it may be seen that the first peak obtained was varying with hole size. Hence a clear conclusion of cavitation could not be derived from this peak irrespective of cage

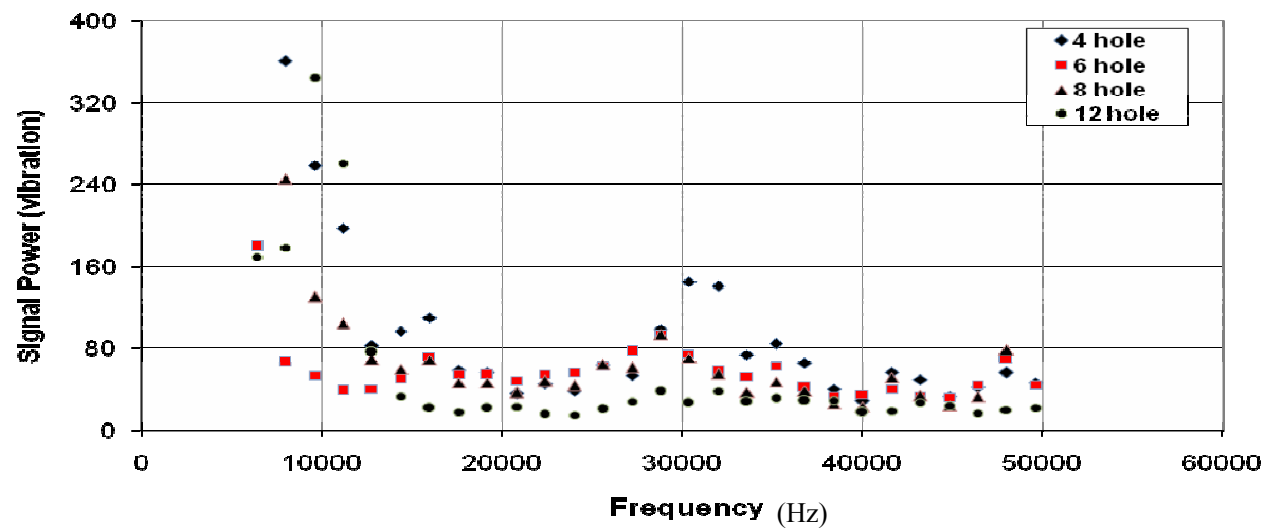

Fig. 9 FFT spectrum for different cage configurations for a typical differential pressure (14 bar, $\sigma=1.074$ ) across test valve

configuration. Second peak was obtained at $30 \mathrm{kHz} . \pm \mathrm{BW}$ for all the configurations tried with $\sigma$ varied from 1.11 to 1.05 . Hence for further analysis of valve cavitation second peak was considered.

From the above figures, it can be seen the relative amplitude increases with increase in pressure drop created by the valve. At the same time, for the same pressure drops, with increase in number of hole, the amplitude of signal decreases.

Figure 10 shows the plot of amplitude of accelerometer signal obtained at $30 \mathrm{kHz}$ for the four of configurations tried. Maximum amplitude was obtained for configuration with 4 holes and minimum for 12 holed configurations. The increment in signal amplitude was more, as the number of holes was increased form 4 to 6 as compared to 6 to 8 or 8 to 12 . For configurations with holes 6 or above, the decrement in the maximum amplitude is not very substantial for maximum differential pressure of 17 bar $(\sigma=1.059)$. 
This decrement in amplitude due to cavitation can be combined with decrease in valve capacity obtained during flow tests. There was a sharp reduction in valve capacity factor, $\mathrm{Cv}$ as the number of holes were decreased from 4 to 6 . This implies that, the intensity of

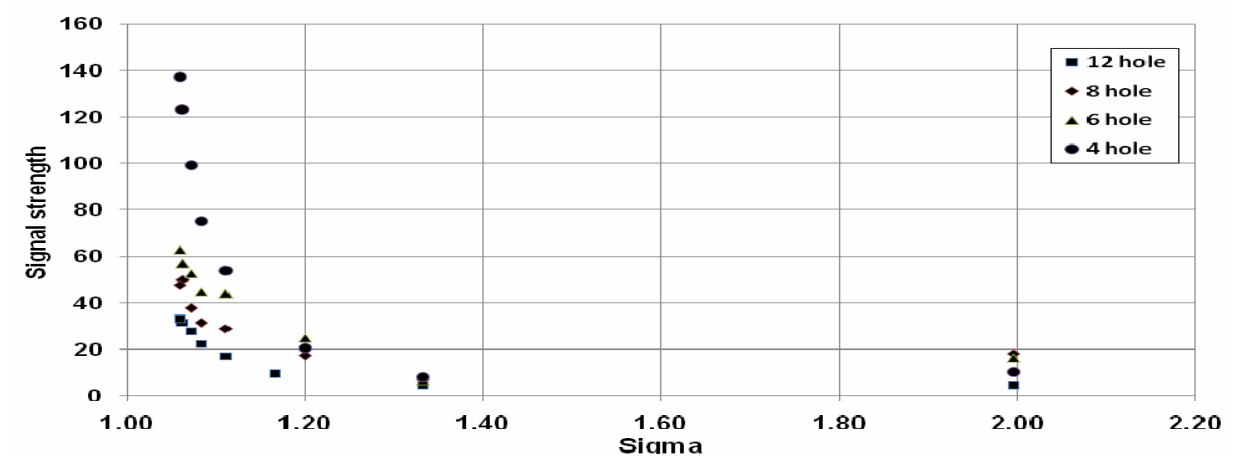

Fig. 10 Plot of signal power at $30 \mathrm{kHz}$ for varying hole configurations

cavitation decreases as the number of holes increased in the cage. This can also be correlated to critical $\sigma$ obtained during overall signal strength analysis.

\section{Conclusions}

A 3" NB control valve with 5 cages designed for cavitation study was studied for flow capacity and extent of cavitation at full open condition of the valve. From the above study following conclusions were arrived at.

1. For area of opening and lift of the valve remaining constant, with increase in no. of opening will decrease valve capacity $(\mathrm{Cv})$ of the valve.

2. As the number of holes increases, the extent of cavitation as measured by dynamic pressure fluctuations and pipe wall vibration decreases.

3. It is possible to predict the onset of cavitation with change in slope of straight line fit of overall signal values obtained for pipe wall vibration and dynamic pressure fluctuations against $\sigma$.

4. FFT analysis of pipe wall vibration and pressure fluctuation revealed that, extent of cavitation can be arrived at, by studying the signal strength at some predominant frequencies. Also it is possible to detect cavitation more precisely with an accelerometer, by measuring the overall vibration with a suitable high pass filter. The lower cut off frequency based on further analysis with different cage configurations.

5. For the same valve, inception of cavitation can be reduced by increasing the number of openings. But increase in number of holes will reduce the flow capacity of the valve. Reduction in severity of cavitation decreases with increase in number of holes in the cage.

\section{References}

(1) Hammitt, F.G., "Cavitation and multiphase flow phenomena", McGraw-Hill International book company, USA, (1980), pp 106-117.

(2) Brennen C.E, “Cavitation \& Bubble Dynamics”, Oxford University Press, (1995), pp 136-144.

(3) Wang, Y.C. and Brennen, C.E., "One-dimensional cavitational flow through a converging diverging nozzle", Journal of Fluids Engineering, vol. 124, no. 9, (1998), pp 166-175.

(4) David, J.A. and Stewart, M., "Prediction of Control valve performance - Part I \& Part II”, Journal of Fluids Engineering, vol. 124, no. 9, (2002), pp 772-779. 
(5) Ramamurthi.K, Nandakumar.K "Characteristics of flow through small sharp edged cylindrical orifices", Flow Measurement \& Instrumentation, Vol. 10, No. 2, (1999), pp $133-143$.

(6) Ramamurthi,K, and Patnaik,SR, "Influence of periodic disturbance on inception of cavitation in sharp edged orifices”, Experiments in fluids, vol. 33, (2003), pp 720- 727.

(7) Takahashi,K, Matsuda,H. and Miyamoto,H, "Cavitation characteristics of Restriction Orifices", Proceedings of $4^{\text {th }}$ international symposium on cavitation -Cav2001, California Institute of Technology, California, June (2001), http://resolver.caltech.edu/CAV2001:sessionB6.005

(8) Tullis, J.P., "Testing valves for cavitation", Proceedings of Conference on Cavitation, the Institution of Mechanical Engineers, London, (1974), pp 45-56.

(9) Tullis, J.P., “Cavitation guide for control valves”, Report No. NUREG/CR-6031-1993, US Nuclear Regulatory commission, (1993), pp 29-44.

(10) Tullis, J.P. and Ball, J.M., "Cavitation data for valves and its application", Proceedings of Conference on Cavitation, the Institution of Mechanical Engineers, London, (1974), pp 55-68.

(11) Cudina, M, “ Detection of cavitation in a centrifugal pump using audible sound", Mechanical systems and signal processing, vol.17(6), (2003), pp 1335-1347.

(12) Gudra, T and Opulinski, K.J, “Applying spectrum analysis \& Cepstrum analysis to examine the cavitation threshold in water and salt solutions", Ultrasonics, Vol. 52, (2004), pp $621-627$. 